\title{
The effect of serum D-dimer levels on the cognitive outcome of patients with moderate traumatic brain injury
}

\author{
Mohammed F.A Ali ${ }^{*}$, Ahmed M.F Ghoul and Mohamed I Refaat
}

\begin{abstract}
Background: Cognitive impairment after traumatic brain injury (TBI) is a main source of morbidity for affected individuals, their family members, and their community. There are numerous serum biomarkers, which are elevated after TBl; one of these is D-dimer. Several studies have related that trauma-related coagulopathy, marked by elevated D-dimer levels, is associated with poor prognosis.

Objective: The aim of this study was to find if there is a correlation between elevated serum levels of D-dimer and impairment of final cognitive outcome in case suffering of Moderate TBI. Patients and method

This is a prospective cohort study with a random sample of 87 patients suffering from moderate TBI. Serum level of D-dimer was requested for all cases after confirming the diagnosis of moderate TBI with post-traumatic GCS 9-12. Head trauma treatment protocols were followed according to each case diagnosis and then at the time of discharge cognitive outcome was assessed for all cases. Neurocognition was assessed by Montreal cognitive assessment-Basic (MoCA-B).
\end{abstract}

Results: Eighty-seven cases were included in this trial with a mean age 28 years. $48.3 \%$ of cases were treated conservatively while the remaining $51.7 \%$ needed surgical interventions for different pathologies. Seventy-five cases had elevated levels of serum D-dimer (86.2\%) whereas only 12 cases have within normal levels (13.8\%). A twofold increase in the serum D-dimer level was found in $41 \%$ of cases, while $34.5 \%$ of cases had three- to sixfold increases, and $10 \%$ of cases had more than sixfold increase. The mean MoCA-B score was 24 points (range 13-27). Correlating the D-dimer levels statistically with the MoCA scores, age, admission and discharge GCS, and durations of hospital stay did not show any statistical significance with any of these variables.

Conclusion: The role played by D-dimer in the pathophysiology of cognitive deficits and its correlation with post moderate TBI cognitive outcome was not proven.

Keywords: D-dimer, Cognitive outcome, Traumatic brain injury, Serum biological markers, Montreal cognitive assessment-Basic

\section{Introduction}

Traumatic brain injury (TBI) remains as a worldwide leading cause of death and disability, representing a major health, economic, and social problem. TBI is considered as the leading cause of death below the age of 45

* Correspondence: dr.mohamedfathy2006@gmail.com

Department of Neurosurgery, Faculty of Medicine, Cairo University, 6 Sray El Manial, Cairo, Egypt and also the cause of death in $25 \%$ of trauma patients. Still in Egypt, we do not have updated statistical records for TBI; however, TBI is thought to represent a major public health problem in Egypt, representing $17.2 \%$ of trauma patients. The most common cause of TBI in Egypt is road traffic accidents, followed by falls and hits by heavy objects [1-3].

Various factors determine the prognosis after TBI. The major factor determining the prognosis after TBI

\section{Springer Open}

(c) The Author(s). 2021 Open Access This article is licensed under a Creative Commons Attribution 4.0 International License, which permits use, sharing, adaptation, distribution and reproduction in any medium or format, as long as you give appropriate credit to the original author(s) and the source, provide a link to the Creative Commons licence, and indicate if changes were made. The images or other third party material in this article are included in the article's Creative Commons licence, unless indicated otherwise in a credit line to the material. If material is not included in the article's Creative Commons licence and your intended use is not permitted by statutory regulation or exceeds the permitted use, you will need to obtain permission directly from the copyright holder. To view a copy of this licence, visit http://creativecommons.org/licenses/by/4.0/. 
is thought to be the primary insult, which is irreversible. However, reversible secondary insults also have a role in determining the outcome of TBI. These secondary insults include cerebral ischemia, intracranial hypertension, systemic hypotension, hyperthermia, and hypoglycemia [4]. TBI patients are exposed to trauma-related coagulopathy due to the release of thromboplastin in high concentrations from the injured brain tissue. This coagulopathy is thought to be one of the prognostic factors of outcome in TBI [5].

One of the common symptoms following moderate TBI is impairment of higher brain cognitive functions. It is thought that resolution of these symptoms occurs after an average of 3 months after trauma; however, it may persist for longer periods in few cases, named persistent post-concussion syndrome. Disturbances of attention, executive functioning, and memory are the most common neurocognitive symptoms following TBI [6].

Cognitive impairment after traumatic brain injury (TBI) is a main source of morbidity for affected individuals, their family members, and their community. Because of the high incidence of physical, neurologic, and psychiatric syndromes following TBI, a full psychiatric assessment of patients should be done before prescription of any treatment for impaired cognition [7].

$\mathrm{D}$-dimer is a fibrin degradation product, introduced in medical practice in the 1990s for diagnosis of deep venous thrombosis, pulmonary embolism, and disseminated intravascular coagulopathy. Increased serum D-dimer concentrations are usually associated with poor outcome after moderate and severe TBI. The use of biochemical markers can be helpful to identify patients at risk of deterioration and hence this draws a better attention for these cases [8].

In this research, we were studying the effect of the serum levels of D-dimer on the prognosis of moderate TBI, and also to identify if there was a relation between serum levels of D-dimer and the final cognitive outcome of these cases.

\section{Method}

This is a prospective cohort study conducted on a sample of 87 cases who presented to the emergency department of Cairo University hospitals following head trauma. Data was collected over a period of 3 months, from January 2018 to September 2018. All patients with signs of moderate TBI, GCS 9-12, all ages, and both sexes were included in this study. Cases with mild or severe TBI, cases with associated thromboembolic comorbidities, and cases on antiplatelet or anticoagulant medications were excluded from this study. Also, cases with a final Glasgow outcome score less than 3 were excluded from this study.
The primary outcome measured was the neurocognitive functions of the patients before final discharge from the emergency neurosurgery unit after finishing their routine management plan, and after retaining their full consciousness and awareness. Several tests are valid for the assessment of neurocognitive functions; we have chosen to use the Arabic version of Montreal cognitive assessment-Basic (MoCA-B) [9].

The Arabic version of MoCA-B is available at http:// www.mocatest.org and was used after permission of publication. It is a one-page 30 points screening test administrated in 10 min to identify cognitive impairment. The total possible score is 30 , a score of 26 or above is considered normal, and a score below 26 indicates cognitive impairment. The MoCA has a high sensitivity (90\%) to detect any mild cognitive impairment with also high specificity (87\%) [9].

Serum concentration of D-dimer was requested for all cases included in this study on admission in our trauma department (mean time between the trauma and withdrawal of D-dimer was $8 \mathrm{~h}$ ). The normal reference range of D-dimer for non-pregnant adults is below $500 \mathrm{ng} / \mathrm{ml}$. Values of serum D-dimer of cases was expressed as "how many folds increase" than the normal range.

We followed the Brain Trauma Foundation guidelines for the treatment of moderate and severe TBI in all out cases [10]. Patients were managed in the neurointensive care unit, intermediate care unit, or the inpatient regular ward according to their clinical state. Surgical interventions were done when indicated.

Outcome assessment was done using the Glasgow outcome scale [11]. Results from cases with final scale scores 3,4 , and 5 (severe, moderate, and mild disability) were selected to be included in the study, while cases with Glasgow outcome score less than 3 (death and vegetative state) were excluded from the study.

\section{Results}

Forty-two cases with moderate TBI presented to the emergency department of Cairo University hospitals during the study period. Twenty-nine cases that had a final Glasgow outcome score of 3 or more were included in this study and their cognitive functions investigated. Cases with a final Glasgow outcome score less than 3 were excluded from the study.

\section{Patient demography}

We included in the study 61 males (70.1\%) and 26 females (29.9\%). Their ages ranged from 18 and 63 years. The mean age was 28 years $(\mathrm{SD}=15)$.

\section{Mode of trauma}

Among the cases included in the study, 48 cases (55.2\%) presented after a road traffic accident (RTA), 21 cases 
(24.1\%) after an isolated trauma to the head, 12 cases (13.8\%) after a fall from height, and 6 cases (6.9\%) after an old trivial head injury.

\section{Clinical presentations}

All cases presented with a history of head trauma and post-concussion syndrome. Seventy-two patients (27.6\%) suffered from weakness (hemiparesis, monoparesis). Sixty-three patients (24.1\%) have raccoon eye, 45 patients (17.2\%) experienced CSF rhinorrhea and otorrhea, whereas 9 patients (3.4\%) experienced bleeding per ear.

\section{Management plan}

Among the cases included in the study, 42 cases (48.3\%) were treated conservatively and 45 cases $(51.7 \%)$ were treated by surgical intervention.

\section{Radiological finding}

Hemorrhagic brain contusion had the highest incidence of occurrence among our cases (27.6\%). Table 1 summarizes the radiological findings in all cases.

\begin{tabular}{|c|c|c|c|c|}
\hline Radiological finding & No. & $\%$ & Management & \\
\hline \multirow[t]{2}{*}{ Contusion } & 24 & $27.6 \%$ & Conservative & 24 \\
\hline & & & Surgical & 0 \\
\hline \multirow[t]{2}{*}{ Compound depressed (CD) fracture } & 15 & $17.2 \%$ & Conservative & 0 \\
\hline & & & Surgical & 15 \\
\hline \multirow[t]{2}{*}{$C D$ and contusion } & 6 & $6.9 \%$ & Conservative & 0 \\
\hline & & & Surgical & 6 \\
\hline \multirow[t]{2}{*}{ Extradural hematoma } & 15 & $17.2 \%$ & Conservative & 0 \\
\hline & & & Surgical & 15 \\
\hline \multirow[t]{2}{*}{ Skull base fracture } & 12 & $13.8 \%$ & Conservative & 12 \\
\hline & & & Surgical & 0 \\
\hline \multirow[t]{2}{*}{ Frontal air sinus fracture } & 9 & $10.3 \%$ & Conservative & 6 \\
\hline & & & Surgical & 3 \\
\hline \multirow[t]{2}{*}{ Chronic subdural hematoma } & 6 & $6.9 \%$ & Conservative & 0 \\
\hline & & & Surgical & 6 \\
\hline
\end{tabular}

Table 1 Summary of radiological findings and management plan

\begin{tabular}{ll}
\hline Management & Number of cases \\
\hline Conservative & $42(48.3 \%)$ \\
Surgical & $45(51.7 \%)$ \\
Total & 87 \\
\hline
\end{tabular}

Table 2 Number of cases according different groups of serum $\mathrm{D}$-dimer concentrations (normal range of serum $\mathrm{D}$-dimer is $0.5 \mu \mathrm{g} / \mathrm{ml}=500 \mathrm{ng} / \mathrm{ml})$

\begin{tabular}{llllll}
\hline D-dimer & $\mathbf{0 - 5 0 0}$ & $\mathbf{- 1 0 0 0}$ & $\mathbf{- 1 5 0 0}$ & $\mathbf{- 2 0 0 0}$ & $\mathbf{- 2 5 0 0}$ \\
\hline No. of cases & $12(13.8 \%)$ & $36(41.4 \%)$ & $15(17.2 \%)$ & $9(13.8 \%)$ & $6(6.9 \%)$ \\
D-dimer & -3000 & -3500 & -4000 & -4500 & -5000 \\
No. of cases & 0 & $3(3.4 \%)$ & $3(3.4 \%)$ & 0 & $3(3.4 \%)$ \\
\hline
\end{tabular}

\section{Serum D-dimer concentrations}

D-dimer was elevated above the normal range $(500 \mathrm{ng} / \mathrm{ml})$ in 75 cases $(86.2 \%)$. The highest percentage of cases (41.4\%) had twofold increases in their serum D-dimers levels. Table 2 summarizes the D-dimer levels of all cases.

\section{Cognitive assessment (MoCA-B results)}

Cognitive assessment for patients who experienced moderate traumatic brain injury included in this study was done prior to discharge after completing either their conservative or surgical management plan during their hospital stay (the mean duration was 3 weeks from the onset of trauma). The highest score achieved was 27 and the least was 13. The mean score was $24(\mathrm{SD}=4)$. Table 3 shows different MoCA$\mathrm{B}$ scores in relation to D-dimer levels.

It is to be noted that the highest serum concentration of D-dimer (5000) was not of the worst MoCA-B score, yet the worst score (13) was found in 6 patients whose D-dimer concentrations were 1613 and 2079.

Statistical studying of the valves did not show any significance between the D-dimer levels and all the studied variables ( $p$ value >0.05). The studied variables were then MoCA-B score, age, admission and discharge GCS, Glasgow outcome score, hospital stay, and CSF leak. We used the "Spearman's rho method" for the detection of significance.

\section{Discussion}

Traumatic brain injury (TBI) is the damage to the brain tissue as sequelae of an external mechanical force, such as rapid acceleration or deceleration, penetration by an object, or direct blow. Brain functions are temporarily or permanently impaired and structural damage may or

Table 3 Case numbers in different MoCA-B scores and its corresponding D-dimer concentrations

\begin{tabular}{lll}
\hline MoCA-B score & No. of cases & D-dimer range \\
\hline 27 & $18(20.7 \%)$ & $545-4520$ \\
26 & $30(34.5 \%)$ & $380-2245$ \\
25 & $27(31 \%)$ & $377-1730$ \\
16 & $3(3.4 \%)$ & $4812-5000$ \\
15 & $3(3.4 \%)$ & $810-830$ \\
13 & $6(6.9 \%)$ & $1613-2079$ \\
\hline
\end{tabular}


may not be detectable with current investigation techniques $[2,12]$.

Disturbed conscious level and post-traumatic amnesia are neurobehavioral trademarks of TBI. According to the classic definition of moderate TBI, there should be loss of consciousness more than $30 \mathrm{~min}$ and more than at least $24 \mathrm{~h}$ of post-traumatic amnesia. Patients may also suffer from agitations and disturbed sleep-wake cycles. Usually, this acute confusional state resolves within several days in uncomplicated cases. However, $65 \%$ of moderate and severe TBI patients have long-term cognitive function impairment interfering with their normal activities and work. Nearly $25 \%$ of moderate and severe TBI patients fail to return to their work in the year following their injury [13].

The primary mechanical injury in TBI triggers a dynamic neuropathologic process ending to a cascade of metabolic, vascular, cellular, and molecular consequences that lead to neurologic deterioration and secondary brain injury. This cascade of events has several serum biomarkers including D-dimer. In this study, we are trying to find a correlation between elevated levels of serum Ddimer and impairment of cognitive functions following moderate traumatic brain injury [14].

This study included 87 cases presenting with moderate TBI. $72.4 \%$ of cases were male and $27.6 \%$ were females. The median age was 28 years, ranging from 18 and 63 years. $55.2 \%$ of cases experienced road traffic accidents, $24.1 \%$ had isolated traumas to the head, $13.8 \%$ had fall from height, and $6.9 \%$ of cases had an old trivial head trauma. These numbers are similar to most of the previously published series of head trauma patients. Middle age males are always predominant in head trauma studies, as these males are more socially active and aggressive than females and older ages [12]. In the study published by Iskhakov et al., falling from height was the most common cause of head injury in $45.2 \%$ of cases and the study published by Subedi et al. in $46.6 \%$ of cases [1]. This difference in the incidence of the mode of trauma can be explained by the driving attitudes and the conditions of the roads in Egypt.

Nearly $60 \%$ of our patients represented within $12 \mathrm{~h}$ of ictus and the rest $40 \%$ presented only after $12 \mathrm{~h}$. In the study published by Subedi et al. [1], 32\% of our patients represented within $12 \mathrm{~h}$ of ictus and the rest $68 \%$ presented only after $12 \mathrm{~h}$.

In our study, $27.6 \%$ of cases suffered from motor weakness, $24.1 \%$ had raccoon eye, and $17.2 \%$ experienced CSF rhinorrhea and otorrhea whereas 3.4\% experienced bleeding per ear. Hemorrhagic brain contusion came first in incidence among our cases, followed by extradural hemorrhages, compound depressed fractures, skull base fractures, and then chronic subdural hemorrhages. Similar results were published in the study Subedi et al. and by Naseri et al. $[1,15]$.

Our study showed D-dimer elevations above the normal range in $86.2 \%$ of cases. The highest percentage of cases (41.4\%) had twofold increases in their serum D-dimers level, followed by a threefold increase in $17.2 \%$ of cases. $13.8 \%$ of cases had normal serum D-dimer levels. These numbers were higher than the incidence found by Subedi et al. in their published study. They stated an increase in the serum D-dimer levels in only $31.1 \%$ of their cases [1]. However, our results were consistent with other studies published by Gomez PA et al. and Harhangi et al., who had increased D- dimer levels in $95.2 \%$ and $84.6 \%$ of cases respectively $[16,17]$.

The mean MoCA-B score among our cases was 24, the highest score was 27, and the lowest score was 13 . $55 \%$ of cases had normal MoCA-B score, denoting normal cognitive functions. Thirty-one percent of cases had borderline MoCA-B score denoting slight affection of their cognitive functions. The remaining $14 \%$ had low MoCA-B scores denoting severe impairment of cognitive functions. We did not detect any statistically significant correlation between D-dimer and MoCA-B scores, or between the $\mathrm{D}$-dimer levels and other variables. This was inconsistent with the results published by Subedi et al., who found a correlation between D-dimer levels and the duration of hospital stay, ICU stay, as well as the Glasgow outcome score [1]. This can be explained by the fact that they were lacking the quantitative assay of D-dimer in their study. Therefore, the role played by Ddimer in the pathophysiology of cognitive deficits and structural abnormalities in moderate TBI is not proved. It takes more thorough and extensive multi-centric studies to be devoted to that purpose. Our study limitations were the lack of a larger number of cases, multicentricity should be taken into consideration, and also a more specific biomarker to TBI should be investigated.

\section{Limitations of the study}

1. The number of cases included shall be larger covering all age groups with stratification.

2. Multi-centricity shall be taken into consideration for more concrete evidence and results.

3. D-dimer is not a specific biomarker or prognostic indicator for traumatic brain injury. Much more specific biomarkers shall be taken into consideration, and this shall raise the idea of much more sophisticated laboratory and imaging investigations to be done and hence the need for more budget to cover. 


\section{Conclusion}

This study included 87 patients who have experienced moderate traumatic brain injury. The majority of the cases had elevated D-dimer levels whereas. These elevated concentrations of such a biomarker have a weak correlation with post-concussion cognitive impairment assessed by MoCA-B. Therefore, the role played by Ddimer in the pathophysiology of cognitive deficits and structural abnormalities in moderate TBI is not proved. It takes more thorough and extensive multi-centric studies to be devoted to that purpose. This could help significantly in expecting, monitoring, and tracing the prognosis of these categories of patients subjected to head trauma.

\section{Abbreviations \\ TBI: Traumatic brain injury; GCS: Glasgow coma scale; GOS: Glasgow outcome scale; CNS: Central nervous system; MoCA-B: Montreal cognitive assessment-Basic; ICU: Intensive care unit; RTA: Road traffic accident; CD fracture: Compound depressed fracture}

\section{Acknowledgements}

Not applicable.

\section{Authors' contributions \\ All authors have read and approved the manuscript. M FA A: Idea of the research, data interpretation and analysis, writing the paper. A M F G: Data collection, data interpretation and analysis, writing the paper. M I R: Supervising data collection interpretation, statistical analysis, writing the paper.}

\section{Funding}

Not applicable

\section{Availability of data and materials}

The datasets and analyzed during the current study are available from the corresponding author on reasonable request

\section{Declaration}

\section{Competing interest}

The authors declare that they have no competing interests

\section{Ethics approval and consent to participate}

This study is approved from the ethical committee of Neurosurgery Department Kasr Alaini Hospital (the reference number is not applicable). The consent obtained from the study participants was verbal as withdrawal of samples was done with the withdrawal of routine labs with no additional risk on study participants.

\section{Consent for publication}

Not applicable

Received: 8 October 2019 Accepted: 15 April 2021

Published online: 24 May 2021

\section{References}

1. Bayir A, Kalkan E, Koçak S, Ak A, Cander B, Bodur S. Fibrinolytic markers and neurologic outcome in traumatic brain injury. Neurol India. 2006;54(4):3635. https://doi.org/10.4103/0028-3886.28106.

2. Montaser T, Hassan A, Ibrahim A. Epidemiology of moderate and severe traumatic brain injury in Cairo University Hospital in 2010. Scand J Trauma Resusc Emerg Med. 2013;21(Suppl 2):A4. https://doi.org/10.1186/17577241-21-S2-A4

3. Faheem S, Hinson H. Pathophysiology and clinical management of moderate and severe traumatic brain injury in the ICU. Semin Neurol. 2015; 35:42-9.
4. Grigorakos L, Alexopoulou A, Tzortzopoulou K, et al. Predictors of outcome in patients with severe traumatic brain injury. J Neurosci Clin Res. 2016;1:1.

5. Kuo JR, Lin KC, Lu CL, Lin HJ, Wang CC, Chang CH. Correlation of a high Ddimer level with poor outcome in traumatic intracranial hemorrhage. Eur J Neurol. 2007;14(10):1073-8. https://doi.org/10.1111/j.1468-1331.2007.01908.x.

6. Mclnnes K, Friesen CL. McKenzie, et al: Mild traumatic brain injury (mTBI) and chronic cognitive impairment: A scoping review. PLOS ONE. 2017;12(4): e0174847. https://doi.org/10.1371/journal.pone.0174847.

7. Arciniegas DB, Anderson CA, Topkoff J, McAllister T. Mild traumatic brain injury: a neuropsychiatric approach to diagnosis, evaluation, and treatment. Neuropsychiatr Dis Treat. 2005;1(4):311-27.

8. DeFazio M, Rammo R, Robles JR, et al. The potential utility of blood-derived biochemical markers as indicators of early clinical trends following severe traumatic brain injury. World Neurosurg. 2014;81(1):151-8. https://doi.org/1 0.1016/j.wneu.2013.01.015

9. Nasreddine ZS, Phillips NA. Bedirian V, at al: The Montreal cognitive assessment, MoCA: a brief screening tool for mild cognitive impairment. J Am Geriatr Soc. 2005;53(4):695-9. https://doi.org/10.1111/j.1532-5415.2005. 53221.x.

10. Brain Trauma Foundation, American Association of Neurological Surgeons, Congress of Neurological Surgeons, Joint Section on Neurotrauma Critical Care, Bratton SL, Chestnut RM, Ghajar J, et al. Guidelines for the management of severe traumatic brain injury. J Neurotrauma. 2007;24(Suppl 1):S1-106.

11. Jennett $B$, Bond $M$. Assessment of outcome after severe brain damage. Lancet. 1975;1(7905):480-4.

12. Mushkudiani NA, Engel DC, Steyerberg EW, Butcher I, Lu J, Marmarou A, et al. Prognostic value of demographic characteristics in traumatic brain injury: results from the IMPACT study. J Neurotrauma. 2007;24(2):259-69. https://doi.org/10.1089/neu.2006.0028.

13. Rabinowitz AR, Levin HS. Cognitive sequelae of traumatic brain injury. Psychiatr Clin North Am. 2014;37(1):1-11. https://doi.org/10.1016/j.psc.2 013.11.004.

14. Sugimoto K, Suehiro E, Shinoyama M, Sadahiro H, Haji K, Fujiyama Y, Kawano R, Nishioka M, Suzuki M. D-dimer elevation as a blood biomarker for detection of structural disorder in mild traumatic brain injury. J Neurotrauma. 2017;34(23):3245-8. https://doi.org/10.1089/neu.2017.5240. Epub 2017 Oct 27

15. Naseri M, Tomasian A, Moghaddas AR. Ccorrelation of CT scan with the level of consciousness in acute head trauma. Iran J Radiol. 2005;2:3-4.

16. Gomez PA, Lobata RD, Ortega JM, et al. Mild head injury: differences in prognosis among patients with Glasgow Coma Scale score of 13 to 15 and analysis of factors associated with abnormal $C T$ findings. Br J Neurosurg. 1996;10(5):453-60. https://doi.org/10.1080/02688699647078.

17. Harhangi BS, Kompanje EJ, Leebeek FW, et al. Coagulation disorders after traumatic brain injury. Acta Neurochir (Wien). 2008;150(2):165-75. https:// doi.org/10.1007/s00701-007-1475-8.

\section{Publisher's Note}

Springer Nature remains neutral with regard to jurisdictional claims in published maps and institutional affiliations.

\section{Submit your manuscript to a SpringerOpen ${ }^{\circ}$ journal and benefit from:}

- Convenient online submission

- Rigorous peer review

- Open access: articles freely available online

- High visibility within the field

- Retaining the copyright to your article

Submit your next manuscript at $>$ springeropen.com 